\title{
La ciencia de los principios y de las causas primeras en el libro primero de la Metafísica
}

\author{
The science of the principles and the first \\ causes in the first book of the Metaphysics
}

\author{
José Antonio GARCÍA-LORENTE
}

Universidad de Murcia

Recibido: 24-02-2015

Aceptado: 03-09-2015

\section{Resumen}

En este trabajo se presenta la concepción aristotélica de la filosofía primera como ciencia de los principios y de las causas primeras según el libro primero de la Metafísica. Para ello, se distinguen tres momentos sucesivos que constituyen el análisis de la naturaleza y la meta que debe alcanzar esta ciencia: 1) la concepción de la sabiduría como ciencia que se ocupa de ciertos principios y causas; 2) la sabiduría como ciencia de los primeros principios y de las causas; 3) la determinación de las cuatro causas primeras como tarea de la filosofía primera. De este modo, se pretende mostrar que la Metafísica de Aristóteles es un intento para explicar las últimas cuestiones, el último porqué, indicando cuatro géneros diferentes de respuesta.

Palabras clave: Aristóteles, causa, filosofía primera, principio.

\begin{abstract}
This paper pretends to show the aristotelian conception of the first philosophy as the science of the principles and the first causes according to the first book of the Metaphysics. In order to do this, three successive moments are distinguished which constitute the analysis of the nature and the goal that this science must attained: 1) the conception of wisdom as a science that deals with certain principles and causes; 2) wisdom as a science of first principles and causes; 3 ) the determination of the four first causes as a task of the first philosophy. In this way, the aim of this work
\end{abstract}


is to show that the Metaphysics of Aristotle is an attempt to explain the ultimate questions, the ultimate why, indicating four different genera of answer.

Keywords: Aristotle, cause, first philosophy, principle.

\section{Breve historia sobre el libro primero de la Metafísica}

Sobre la autenticidad, composición y el lugar que ocupa este libro en la Metafísica de Aristóteles se ha debatido mucho entre los especialistas. En efecto, sobre el primer libro que forma parte del texto que nos ha sido transmitido con el título de Metafisica se han vertido serias dudas sobre su autenticidad en la Antigüedad y en el Medioevo, como han puesto de manifiesto los especialistas que participaron en el IX Symposium aristotelicum en 1981, dedicado a la autenticidad de las obras que forman parte del actual Corpus aristotelicum ${ }^{1}$. Esto puede venir propiciado por la propia naturaleza del libro primero de la Metafísica, que, como sabemos, es anterior a un libro también primero, en este caso, referido con la letra alfa minúscula (" $\alpha ")^{2}$.

El testimonio más antiguo de estas dudas procede del comentador Asclepio (s. VI d. C,), el cual indica que el libro "A", es decir, el libro que se ha transmitido como primero, venía atribuido erróneamente a Pasicles de Rodas, sobrino de Eudemo $^{3}$. La atribución de este libro a Pasicles es manifiesta también en un escolio que aparece entre el final del primer libro y el inicio del segundo, sobre el margen de la hoja, en el manuscrito Parisinus graecus 1853 (indicado por los editores con la sigla E), que es justamente el manuscrito más antiguo de la Metafísica de Aristóteles (s. X). En este lugar, se dice más o menos lo siguiente: "Este libro, según la mayor parte de los críticos, es de Pasicles de Rodas" 4 .

\footnotetext{
${ }^{1}$ Moraux, P., Wiesner, J. (ed.), Zweifelhaftes im Corpus Aristotelicum. Studien zu einigen Dubia. Akten des 9. Symposium Aristotelicum, Walter de Gruyter, Berlin 1983.

2 Para la exposición de este apartado sigo fundamentalmente el texto de Enrico Berti y Cristina Rossitto, Aristotele. Il libro primo della «Metafisica», Laterza, Roma-Bari 1993, pp. 14-19.

3 Ascl., in Metaph., 4, 21-22. Asclepii in Aristotelis Metaphysicorum libros A-Z commentaria, edidit M. Hayduck, Berolini 1888 (CAG 6, 2), apud Berti, E., y Rossitto, C., Aristotele. Il libro primo della «Metafisica», p. 14.

4 Durante mucho tiempo se ha pensado que este escolio se refiere al libro II, es decir, alfa minúscula, el libro que viene inmediatamente después en el mencionado manuscrito. Sin embargo, los estudios más recientes han puesto de manifiesto que dicha anotación está referida al libro I, o sea, a "A". Cfr. Berti, E., "Note sulla tradizione dei primi due libri della «Metafisica» di Aristotele", en Elenchos, 3, 1982, pp. 5-38 (publicado también en: Nuovi studi aristotelici. II. Fisica, antropologia e metafisica, Morcelliana, Brescia 2005, pp. 241-265.); Bernardinello, S., Gli scoli alla «Metafisica» di Aristotele nel f. 234r del «Parisinus Graecus 1853» (E), in Moraux-Wiesner (herausgegeben), Zweifelhaftes im Corpus Aristotelicum, pp. 39-54; G. Vuillemin-Diem, Anmerkungen zum Pasikles-Bericht und zu
} 
Para identificar quién pudo ser el autor que atribuyó a Pasicles de Rodas la autoría de este libro I, debemos remitirnos una vez más a Asclepio. Éste cuenta que algunos explican la transmisión del texto de la Metafísica afirmando que Aristóteles mismo envió una copia a su discípulo Eudemo y que éste no la publicó debido a su extensión. Después de la muerte de Aristóteles, algunas partes de la obra se extraviaron y sus sucesores la completaron añadiendo partes de otros tratados y adaptándolas como pudieron 5 .

Esta historia explica cómo en la Metafisica que había en Rodas -probablemente la única copia que existía antes de la edición de Andrónico-y que debía comenzar con el libro "A", alguno, esto es, los sucesores de Aristóteles o de Eudemo, podría haber insertado el libro " $\alpha$ ". Cuando Andrónico se encontró esta obra con dos libros primeros, o sea, " $\mathrm{A}$ " y " $\alpha$ ", conservó ambos, pero haciendo entender a través de la numeración asignada que uno de ellos no debía de ser de Aristóteles. En cualquier caso, el hecho es que Andrónico no dudó, como hubiera sido natural, de la autenticidad de " $\alpha$ ", sino de " $A$ ", probablemente, considerando que los sucesores de Eudemo, artífices de la confusión, habrían introducido en la Metafisica de Aristóteles un libro que fue escrito precisamente por uno de ellos, esto es, Pasicles de Rodas. Y este es, en efecto, el origen de la noticia según la cual el libro "A" se atribuye a Pasicles, noticia referida por Asclepio y presente en el manuscrito E.

Sin embargo, la conclusión de Andrónico no fue considerada como cierta por ninguno en la Antigüedad. Ya su contemporáneo Nicolás Damasceno, que escribió un tratado sobre la filosofía de Aristóteles añadiendo un compendio de la Metafisica, resume el libro "A" y el libro " $\alpha$ ", considerando ambos como libros auténticos de Aristóteles 6 . Del mismo modo, Alejandro de Afrodisias, Siriano y Asclepio, o sea, prácticamente todos los comentadores antiguos de la Metafísica no dudaron de la autenticidad del libro I.

Pero vuelven a suscitarse dudas sobre la autenticidad del libro "A" en el Medioevo, con los árabes. En este caso, Al-Farabi (s. IX), en su análisis de la Metafísica, resume el primer libro, pero está referido claramente a " $\alpha$ " y no a "A", dando indicios a pensar que no consideraba "A" como parte de la Metafísica. También, el Gran comentario de Averroes a la Metafísica compete sobre todo a " $\alpha$ " y después únicamente trata la segunda mitad de "A" (a partir de 987a 5-6), aunque bien es cierto que esto puede venir justificado por la ausencia de este libro en las traducciones árabes que Averroes trabajó 7 . Para explicar este fenómeno, Alberto

Echtheitszweifeln am grösseren und kleineren Alpha in Handschriften und Kommentaren, in MorauxWiesner (herausgegeben), Zweifelhaftes im Corpus Aristotelicum, pp. 157-192.

5 Ascl., in Metaph., 4, 4-16, apud Berti, E., y Rossitto, C., Aristotele. Il libro primo della «Metafisica», p. 15.

6 Cfr. Drossaart Lulofs, H. J., Nicolaus Damascenus on the Philosophy of Aristotle, E. J. Brill, Leiden 1969, pp. 74-76.

7 Cfr. Munk, S., Mélanges de philosophie juive et arabe, Vrin, Paris 1955, pp. 434-435; Bouygues, M., 
Magno señala explícitamente que, según la tradición, el libro I de la Metafísica, el que comienza con la famosa frase "todos los hombres desean por naturaleza saber", es decir, el propio libro "A", lo habría añadido Teofrasto 8 .

Probablemente, esta tradición procede de otro escolio conservado también en la manuscrito E, al final de la Metafísica de Teofrasto, que se encuentra inmediatamente después de la de Aristóteles indicando que "este libro" viene mencionado por Nicolás Damasceno en su compendio a la Metafísica de Aristóteles como obra de Teofrasto 9 . Ahora bien, la confusión surge porque, al parecer, en algunas ediciones antiguas de las obras de Aristóteles, la Metafísica de Teofrasto precedía a la Metafísica de Aristóteles, y, por consiguiente el escolio colocado al final de la obra de Teofrasto aparece referido al libro I de la obra aristotélica, que seguía inmediatamente después ${ }^{10}$.

Respecto a la cronología y comenzando con el estudioso Werner Jaeger, quien inaugura el método histórico genético, partiendo del supuesto de que los escritos conservados contienen las huellas de los diferentes estadios de una evolución, se estableció que el libro "A" es un texto temprano. Así pues, el contenido de este libro se correspondería con un primer intento por parte de Aristóteles para definir la sabiduría y que, posteriormente, se integraría como introducción para un proyecto más sofisticado de la ciencia del ente en cuanto ente. En concreto, el influyente Aristóteles de Jaeger en el capítulo 4 establece que Metafisica A, 1-2 no es más que una versión abreviada del Protréptico y, por consiguiente, están esencial o íntegramente tomados de él11.

Sin embargo, esta interpretación del texto aristotélico ha sido rechazada por unanimidad por parte de los participantes en el XVIII Symposium Aristotelicum que tuvo lugar en Lovaina, los días 7-13 de julio de 200812. Giuseppe Cambiano, por ejemplo, reconoce que muchos argumentos presentados en los primeros capítulos de la Metafísica pueden ser asimilados en el contexto del Protréptico, pero otros no, como pueden ser: las distinciones entre empeiría y téchnê, el conocimiento de los

Averroès, Grand Commentaire de la Métaphysique, Beyrouth 1952, pp. CXXVIII-CXXIX e CXLIXCL; Darms, G., Averroes, In Aristotelis librum II Metaphysicorum Commentarius, Freiburg-Schweitz 1966, pp. 12-14.

8 Albertus Magnus, An. Post., I, tr. II, c. 1. Apud, Berti, E., y Rossitto, C., Aristotele. Il libro primo della «Metafisica», p. 16.

9 El escolio es referido por W. D. Ross y F. H. Fobes en su edición de la Metafísica de Teofrasto. Oxford 1929, p. 38. Apud Berti, E., y Rossitto, C., Aristotele. Il libro primo della «Metafisica», p. 17. 10 Este equívoco está también presente en una traducción latina de la Metafisicica de Aristóteles, contenida en la manuscrito Laurentianus Latinus XII sin. 7 (del siglo XIV). Berti, E., y Rossitto, C., Aristotele. Il libro primo della «Metafisica», p. 17.

11 Cfr. Jaeger, W., Aristoteles, de la versión española de José Gaos Aristóteles: Bases para la historia de su desarrollo intelectual, FCE, México 1946, pp. 85-88.

12 Carlos Steel, Oliver Primavesi (editors), Aristotle's Metaphysics Alpha, Oxford University Press, Oxford 2012. Ver el prefacio, pp. v-vi. 
particulares y el conocimiento de los universales, o entre el conocimiento del "que" y el conocimiento del "por qué". En cualquier caso, el hecho de que se encuentren paralelismos lingüísticos o conceptuales en ambas obras no prueba definitivamente la contemporaneidad o dependencia de un texto sobre el otro13.

Finalmente, merece la pena mencionar que en las actas publicadas del Symposium se ofrece la última edición crítica del libro Alpha, teniendo en cuenta toda la tradición manuscrita, las traducciones medievales y la tradición indirecta, ofreciendo nuevos avances sobre la relación de las dos tradiciones de los textos ( $\alpha$ y $\beta$ ), llevada a cabo por Oliver Primavesi, profesor catedrático de Griego en Ludwig-Maximilians-Universität München ${ }^{14}$.

\section{La ciencia de ciertos principios y causas}

El primer libro de la Metafísica está convencionalmente dividido en diez capítulos. En líneas generales, este libro puede dividirse en dos partes principales. La primera comprende los capítulos 1-2 y contiene una discusión sobre la naturaleza de la sabiduría, que se define como conocimiento de las causas primeras; la segunda parte estaría formada por los capítulos 3-10, y es una verificación histórica de la validez de la doctrina de los cuatro géneros de causas formulada en la Física.

En el primer capítulo, una vez que se ha puesto de manifiesto que el ejercicio del conocimiento pertenece a la misma naturaleza del género humano ("todos los hombres desean por naturaleza saber"15), Aristóteles distingue varias formas de conocimiento: la percepción ( $\alpha$ ľ $\sigma \theta \eta \sigma ા \varsigma)$, la imaginación $(\varphi \alpha ́ v \tau \alpha \sigma \mu \alpha)$, el recuerdo

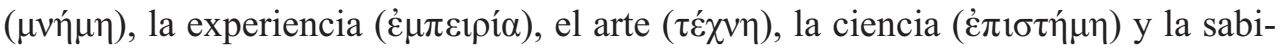
duría ( $\left.\sigma \circ \varphi \varphi^{\alpha} \alpha\right)$. Las características de cada uno de estos modos de conocimiento se hacen explícitas a lo largo de todo el capítulo, discutiendo cuál es la naturaleza del

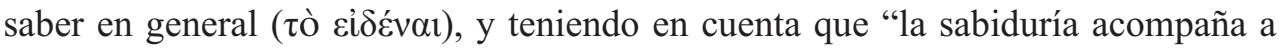
cada uno en mayor grado según el nivel de su saber" 16.

Estos modos de conocimiento comprenden fundamentalmente tres grados del conocimiento, dispuestos en orden progresivo. En primer lugar, percepción, imaginación y recuerdo son tres modos de conocimiento que, en cuanto a su contenido,

\footnotetext{
13 Cfr. Cambiano, G. "The desire to know" en Aristotle's Metaphysics Alpha, pp. 39-42.

14 Primavesi, O., "Aristotle, Metaphysics A. A New Critical Edition with Introduction”, en Aristotle's Metaphysics Alpha, pp. 385-516.

15 Aristóteles, Metafísica, I, 1, 980a 21. Para las citas de la Metafisica utilizo la traducción al español de Tomás Calvo Martínez, Gredos, Madrid 1994, aunque en algunos casos la modifico por preferencias estilísticas. La edición crítica del texto griego se corresponde con la de W.D. Ross, Aristotle's Metaphysics. A revised text with Introduction and Commentary, 2 vols., Oxford Clarendon Press, 1924. Reprinted lithographically, Oxford University Press, 1948, 1953 (with corrections) 1958.

16 Aristóteles, Metafísica, I, 1, 981a 26-27.
} 
pueden ser asimilables entre ellos ${ }^{17}$; en segundo lugar, la experiencia; y finalmente, el arte o la ciencia que, de momento, Aristóteles caracteriza del mismo modo y a cuya distinción nos remitirá a la Ética18.

De entre estas formas de conocimiento, Aristóteles identifica en un primer momento el saber y el conocer con la experiencia, teniendo en cuenta que la sabiduría pertenece a cada uno según el grado de saber, y que la percepción no es sabiduría ${ }^{19}$. Pero a continuación, Aristóteles identifica el saber con el arte, en virtud de aquello que le diferencia de la experiencia. La diferencia está en que la experiencia es conocimiento de las cosas singulares ( $\tau \hat{\omega} v \kappa \alpha \theta^{\prime}$ Ě $\left.\kappa \alpha \sigma \tau o ́ v\right)$, y el arte, de las universales ( $\tau \hat{\omega} v \kappa \alpha \theta \dot{\lambda} \lambda \mathrm{ov})$. Esta distinción se especifica mediante el ejemplo que Aristóteles aduce: "En efecto, el tener la idea de que a Calias tal cosa le vino bien cuando padecía tal enfermedad, y a Sócrates, e igualmente a muchos individuos, es algo propio de la experiencia; pero la idea de que a todos ellos, no delimitados como un caso específicamente idéntico, les vino bien cuando padecían tal enfermedad (por ejemplo a los flemáticos o biliosos o aquejados de ardores febriles), es algo propio del arte" 20 . Aquí, la peculiaridad de la experiencia consiste en su referencia a un caso singular, la enfermedad de Calias, la de Sócrates y la de otros, sin considerar si todos estos casos son la única y misma enfermedad. En cambio, la característica del arte consiste precisamente en considerar esta única enfermedad, presente en todos los casos y, por consiguiente, universal. De este modo, Aristóteles asocia el conocimiento de lo singular con la experiencia y el conocimiento de lo universal con el arte.

Poco después, Aristóteles dice lo siguiente: "Pero no es menos cierto que pensamos que el saber y el conocer se dan más bien en el arte que en la experiencia y tenemos por más sabios a los hombres de arte que a los de experiencia, como que la sabiduría acompaña a cada uno en mayor grado según su saber. Y esto porque los

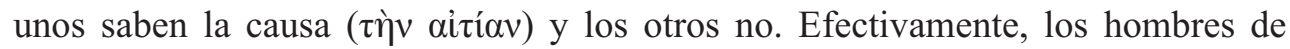
experiencia saben lo que sucede ( $\tau$ ò ö $\tau$ ), pero no el porqué ( $\tau$ ò $\delta$ เó $\imath$ ), mientras que

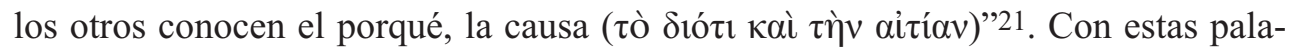
bras, el Estagirita sigue delimitando las diferencias entre arte y experiencia, poniendo de manifiesto que el primero supone un grado mayor de conocimiento que el segundo. La razón reside en que los que poseen el arte conocen la causa y los exper-

\footnotetext{
${ }_{17}$ La imagen es la permanencia de aquello que procede de la percepción (An. post., II, 19, 99b 35-37), mientras que el recuerdo es la conservación de la imagen, reconocida como imagen del objeto (De mem., I, 451a 14).

18 Aristóteles, Metafisica, I, 1, 981b 25-26. Cfr. Eth. Nic., VI, 3-7, 1139b14 - 1141b 8.

19 Aristóteles indica más adelante: “Además, no pensamos que ninguna de las percepciones ( $\tau \hat{\omega} v$

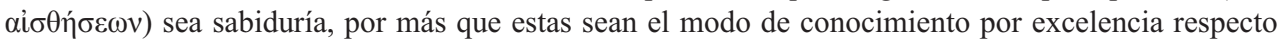
de los casos individuales". Metafísica, I, 1, 981b 10-11.

${ }^{20}$ Aristóteles, Metafísica, I, 1, 981a 7-12.

21 Aristóteles, Metafísica, I, 1, 981a 24-30.
} 
tos no, esto es, los expertos saben lo que sucede, pero no el porqué, mientras que los que poseen arte conocen el porqué y la causa 22 .

Pues bien, una vez establecido que el arte supone un grado superior de conocimiento frente a la experiencia, y establecidas las diferencias entre las ciencias prácticas y las ciencias teoréticas, como conclusión del discurso sobre la caracterización del saber en general, Aristóteles sostiene lo siguiente:

En la Ética está dicho cuál es la diferencia entre el arte y la ciencia y los demás del mismo género; la finalidad que perseguimos al explicarlo ahora es ésta: mostrar cómo todos opinan que lo que se llama sabiduría se ocupa de las causas primeras y de los prin-

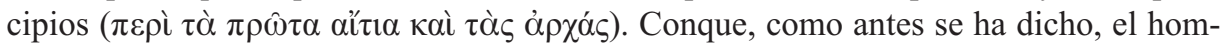
bre de experiencia es considerado más sabio que los que poseen sensación del tipo que sea, y el hombre de arte más que los hombres de experiencia, y el director de la obra más que el obrero manual, y las ciencias teoréticas más que las productivas. Es obvio,

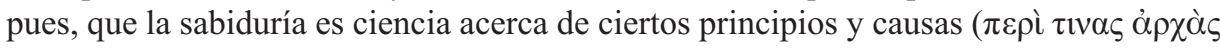
кaì aĩías) $)^{23}$.

Aquí Aristóteles pone de manifiesto, en primer lugar, el objetivo de este primer capítulo: hacer una clasificación de las distintas formas de conocimiento -teniendo en cuenta que la sabiduría acompaña a cada uno según el grado de saber-, para mos-

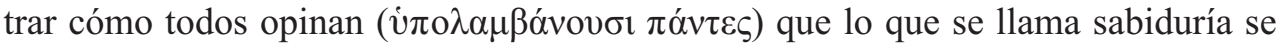

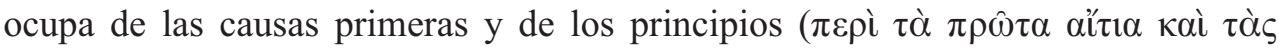

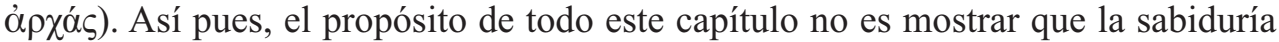
versa sobre las causas primeras y principios, sino mostrar que todos opinan que la sabiduría se ocupa de estos. A mi modo de ver, Aristóteles está presentando una opinión reputada por todos, esto es, un éndoxon, que consiste precisamente en la afirmación de que la sabiduría se ocupa de las causas primeras y de los principios 24 . Por consiguiente, esta referencia a las causas consideradas como primeras, argumento que Aristóteles no ha considerado por el momento y que constituye la conclusión a la que llegará en el capítulo siguiente, es la opinión que todos tienen acerca de qué es la sabiduría, a partir de la consideración de los diversos modos de conocer y teniendo en cuenta que la sabiduría acompaña a cada uno en mayor grado según el nivel de su saber 25 .

\footnotetext{
22 Como se puede apreciar, aquí aparece por primera vez y en el mismo momento dos de los términos

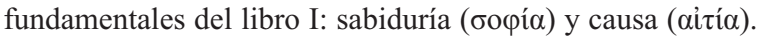

23 Aristóteles, Metafísica, I, 1, 981b 25 - 982a 2.

24 Como es sabido, Aristóteles utiliza el método dialéctico que parte de la consideración de las opiniones más reputadas o autorizadas. Sobre esta cuestión, López Molina-Niñirola, José Miguel, Recepción y significado del uso científico de la dialéctica aristotélica, Pensamiento. Revista de Investigación e Información Filosófica, vol. 71, n. 269 (2015), pp. 1261-1276.

25 Algunos autores han eliminado el término "primeras" ( $\pi \rho \hat{\omega} \tau \alpha)$ de la línea 28 (Wirth), porque hace más comprensible el orden del razonamiento en los capítulos 1-3, ya que el argumento sobre las cau-
} 
Inmediatamente después, Aristóteles resume los resultados adquiridos a lo largo del capítulo, subrayando que el grado superior de conocimiento es más saber que el inferior, pues, como él mismo había dicho, "la sabiduría acompaña a cada uno en mayor grado según (el grado de) su saber"26. En este sentido, el que posee experiencia es más sabio que el que posee solamente la percepción y el que posee el arte es más sabio que el que posee solamente la experiencia. Si además, el arte se distingue de la ciencia, en cuanto que el primero tiene como fin la producción, mientras que el segundo tiene como fin el mismo conocimiento, es evidente que la sabiduría coincide más propiamente con la ciencia y, como toda ciencia, la sabiduría es cono-

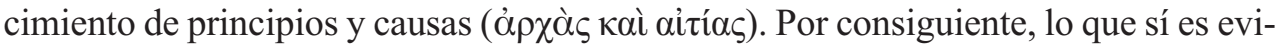
dente es que la sabiduría es ciencia acerca de ciertos principios y causas; que estos sean primeros tendrá que ser demostrado (aunque ya sabemos que todos opinan que sí), y, precisamente, es lo que Aristóteles emprenderá en el siguiente capítulo.

\section{La ciencia de los principios y causas primeras}

Aristóteles ha introducido un grado más en el nivel del conocimiento: la sabiduría. A partir de este momento, en el segundo capítulo, Aristóteles mostrará con qué ciencia coincide la sabiduría, es decir, de qué causas y de cuáles principios se debe ocupar la sabiduría. Pues bien, el segundo capítulo comienza así: "Puesto que andamos a la búsqueda de esta ciencia, habrá de investigarse acerca de qué causas y qué principios es ciencia la sabiduría ( $\left.\sigma 0 \varphi^{\prime} \alpha\right)^{\prime \prime 27 . ~}$

Aristóteles se pregunta lo siguiente: la ciencia que se está buscando, la que debe ser expuesta en este tratado o en este curso, ¿qué ciencia es? ¿De qué causa es ciencia? Como ha señalado Enrico Berti, sabiduría ( $\sigma \circ \varphi i ́ \alpha)$ era una palabra de uso común en la Grecia antigua y no indicaba una forma de saber particular. Indicaba, en cualquier contexto, el grado de saber más alto o más elevado. Así pues, considerando todas las ciencias o artes posibles, lo que Aristóteles se está cuestionando es

\footnotetext{
sas de las que se ocupa la sabiduría como "primeras" todavía no ha sido tratado por Aristóteles. De esta opinión es también Reale, G., Introduzione, traduzione e commentario della Metafisica di Aristotele, Bompiani, Milano 2004, p. 707. Zanatta en su comentario considera que se puede leer como una especie de programa o de resultado final al que se dirige la investigación: Aristotele, Metafisica, Introduzione, traduzione e note di Marcello Zanatta (vol. I), BUR, Milano 2009, p. 363. En el análisis de Giuseppe Cambiano se sostiene que la aparición de la noción "primeras causas" crea perplejidad, y es todavía hoy una cuestión abierta. El problema aumenta sobre todo cuando, a continuación, el texto dice explícitamente que la sabiduría es ciencia sobre ciertas causas y principios, pero no las primeras causas y principios: Cambiano, G. "The desire to know" en Aristotle's Metaphysics Alpha, pp. 38-39. Sarah Broadie, por su parte, considera que las líneas 25-29 son un añadido posterior del propio Aristóteles, "A Science of First Principles", en Aristotle's Metaphysics Alpha, p. 43.

26 Aristóteles, Metafisica, I, 1, 981a 27.

27 Aristóteles, Metafísica, I, 2, 982a 4-6.
} 
lo siguiente: de entre todas las artes, ciencias y disciplinas de este tipo, ¿cuál de esas merece el nombre de sabiduría, por ser la ciencia más alta, la ciencia que nos permite conocer más, que nos da explicaciones más profundas y más completas?28. Pues, resulta evidente, ha dicho Aristóteles, que "la sabiduría es una ciencia sobre ciertos principios y causas" 29 . Ahora bien, ¿cuáles son estos "ciertos principios" y estas "ciertas causas"?

La ciencia buscada era aquella que se ha definido en el capítulo primero como sabiduría, que, en cuanto ciencia, se ocupa de causas y de principios en el sentido de conocerlos. Por consiguiente, la indagación que se emprende a partir de este momento consiste en la determinación de cuáles sean estas causas y estos principios. Para responder a esta pregunta, Aristóteles sigue su método característico, que consiste en considerar las opiniones más difundidas acerca del argumento para examinarlas críticamente. En este caso, el Estagirita examina las diferentes concepciones que se tienen del sabio, que, como sabemos, son seis: 1) sabe ( $\dot{\varepsilon} \pi i ́ \sigma \tau \alpha \sigma \theta \alpha)$ todas las cosas, pero no cada una de ellas individualmente; 2) conoce las cosas más difíciles, es decir, las más alejadas a los sentidos; 3 ) conoce las causas con más rigor; 4) sabe enseñarlas mejor; 5) la ciencia que se elige porque tiene el fin en sí misma es sabiduría en mayor grado que la que se escoge porque tiene el fin en otro; 6) la ciencia que manda ( $\dot{\rho} \rho \chi \iota \kappa \omega \tau \dot{\varepsilon} \rho \alpha v)$ es sabiduría en mayor grado que la subordinada ${ }^{30}$.

Dejando de lado el análisis aristotélico de cada una de las opiniones acerca de la sabiduría y de lo que caracteriza al sabio, Aristóteles concluye del siguiente modo: "Así pues, por todo lo dicho, el nombre en cuestión [el nombre de бopía] corresponde a la misma ciencia. Ésta, en efecto, ha de estudiar acerca de los prime-

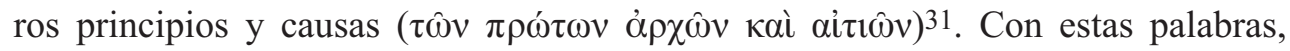
Aristóteles está diciendo que la sabiduría es aquella ciencia que posee en mayor medida el carácter específico del saber. La sabiduría es, por tanto, un saber de los primeros principios y causas.

\section{a) Definición de principio}

El tratamiento aristotélico más amplio dedicado a los principios es el libro I de la Física, considerado por numerosos intérpretes como un tratado en sí mismo, relativamente independiente del resto de la obra, e identificado con la obra "Sobre los principios" ( $\pi \varepsilon \rho \grave{\alpha} \dot{\alpha} \rho \chi \hat{\omega} v)^{32}$. El argumento de este libro consiste en la determi-

\footnotetext{
28 Berti, E., Struttura e significato della Metafisica di Aristotele, Edusc, Roma 2006, p. 36.

29 Aristóteles, Metafísica, I, 1, 982a 1-3.

30 Aristóteles, Metafisica, I, 2, 982a 6-21.

31 Aristóteles, Metafísica, I, 2, $982 \mathrm{~b} 9$.

32 Cfr. Calvo Martínez, J. L., "Introducción” a Aristóteles, Física, CSIC, Madrid 1996, pp. XXXIXXXIX.
} 
nación de los principios del entero mundo de la experiencia y, por esta razón, este libro puede considerarse como expresión de un punto de vista más metafísico que físico 33 .

La cuestión sobre los principios es considerada por Aristóteles como condición indispensable para constituir un auténtico saber, es decir, no simplemente un mero conocimiento de la realidad, sino una verdadera y propia ciencia. Como se menciona en el mismo inicio del libro, tener ciencia de algo significa conocer los principios y las causas, porque "solo creemos poseer conocimiento de cada cosa precisamente cuando reconocemos las causas primeras y los principios primeros" 34 . El método aplicado para el conocimiento de los principios está explícitamente indicado por Aristóteles cuando dice que es preciso proceder "desde lo más cognoscible y claro para nosotros", es decir, del mundo de la experiencia, "hasta lo más claro y cognoscible por naturaleza", o sea, las condiciones de la cognoscibilidad del mundo de la experiencia: los principios 35 .

Después de la crítica a los eleatas, Aristóteles parte de la observación de que todos los filósofos precedentes ponen como principios los contrarios, para concluir que él también está de acuerdo 36 . Y añade inmediatamente después: "Pues los principios no deben proceder ni de otros principios, ni uno del otro, y todas las cosas deben proceder de ellos. Y esto corresponde a los principios si son primeros: el que, por ser primeros, no proceden de otros, ni tampoco proceden uno del otro por ser contrarios" 37 . Con estas palabras, Aristóteles especifica el significado del término principio como aquello desde lo cual todo deriva, señalando sus características, esto es, no derivar de otros principios (en cuanto son primeros), ni tampoco pueden derivar entre ellos el uno del otro.

A continuación, a partir del capítulo 6, Aristóteles comienza propiamente la exposición positiva de los principios que, como sabemos, se constituyen por los

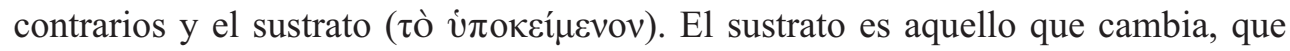
pasa de un contrario a otro, y en las substancias es la materia (iّ $\lambda \eta)$ : el bronce o la piedra son el sustrato de la estatua. Los contrarios son la privación ( $\sigma \tau \varepsilon ́ \rho \eta \sigma ı \varsigma)$ : el oro todavía no es estatua, y la forma ( $\varepsilon \hat{i} \delta o \varsigma$ ), es decir, la determinación positiva que la materia asume ${ }^{38}$. Por tanto, los principios son: materia, forma y privación. La

33 Cfr. Mansion, A., "Introduction" à la physique aristotélicienne, Lovain-Paris, Publications Universitaires de Louven, 1945 (I ed. 1913); Aubenque, P., Le problème de l'être chez Aristote, Paris 1962 [El problema del ser en Aristóteles, Madrid 1974]; Wieland, W., Die aristotelische Physik, Göttingen, Vandenhoeck \& Ruprecht, 1970.

34 Aristóteles, Física, I, 1, 184a 12-14. Cito por la traducción de José Luis Calvo Martínez, CSIC, Madrid 1996.

35 Aristóteles, Física, I, 1, 184a 15-21.

36 Aristóteles, Física, I, 5, 188a 19-27.

37 Aristóteles, Física, I, 5, 188a 27-30.

38 Aristóteles, Física, I, 7, 190b 23-29. 
materia no se constituye como el contrario de la forma, sino como sustrato. Estos principios son los principios constitutivos de la propia realidad, y por consiguiente, son principios inmanentes, es decir, elementos ${ }^{39}$.

A la determinación positiva de estos principios se puede añadir la definición de los términos principio y elemento, contenidos en los capítulos 1 y 3 del libro Delta de la Metafísica. En este lugar, Aristóteles define el término principio en general como "lo primero a partir de lo cual algo es, o se produce, o se conoce" (principio de ser o existir, del cambio y del conocimiento), precisando que algunos principios son internos y otros externos 40 . Así pues, principio es lo primero en el orden de una sucesión, esto es, el inicio espacial o temporal a partir del cual algo comienza: el inicio de una vía, de un movimiento o de un discurso. También es lo primero que da origen a algo: los cimientos de la casa, el padre del hijo. Y finalmente, principio es lo primero a partir de lo cual algo se conoce: las premisas de las demostraciones ${ }^{41}$. El elemento, a su vez, es "lo primero de lo cual algo se compone, siendo aquello inmanente y no pudiendo descomponerse, a su vez, específicamente en otra especie distinta"42. La distinción entre principios, externos e internos o elementos, introduce un nuevo tipo de principio, que ya no es elemento. Este descubrimiento completa la doctrina aristotélica de los principios-elementos, transformándola, como veremos, en la doctrina de las cuatro causas primeras.

\section{b) Definición de causa}

La etimología del término griego aitía ha sido objeto de debate en los últimos años, pero no hay ninguna duda de que estaba referido originariamente a conceptos como acusación, imputación o culpabilidad, haciendo referencia a la idea de ser culpable o responsable de algo. Posteriormente y con la utilización de este término por parte de los primeros filósofos, se han añadido los sentidos de causa, razón, etc.

\footnotetext{
${ }^{39}$ Aristóteles, Física, I, 7, 190b 29 191a 7. Cfr. Aristóteles, Metafísica, XII, 2, 1069b 9ss. Cfr. Berti, E., Aristotele. Dalla Dialettica alla filosofia prima, Bompiani, Milano 2004, pp. 366-373.

40 Aristóteles, Metafisica, V, 1, 1013a 17-19.

41 Cfr. Aristóteles, Metafísica, V, 1.

42 Aristóteles, Metafísica, V, 3, 1014a 26-27.

43 Recientemente se ha producido un nuevo renacimiento sobre la cuestión de la causalidad, en donde la teoría aristotélica protagoniza un papel fundamental. Se ha publicado un volumen de un coloquio internacional que tuvo lugar en París los días 16-18 de septiembre de 2010, que forma parte de un proyecto franco-brasileño, entre el Centre Léon Robin y la USP de Sao Paulo, que lleva por título "Aristote: causes et actions" (2008-2010), y otro volumen que forma parte de un proyecto brasileñoitaliano sobre la noción de causalidad en el mundo griego titulado "AITIA. Dépendance causale, responsabilité et nécessité dans la pensée antique" (2011-2013). Cfr. Natali, C. Viano, C. Zingano, M., (a cura di), Aitia I. Les quatre causes d'Aristote. Origines et interprétation. Leuven, Editions Peeters SA, Leuven Belgium 2013; Natali, C. Viano, C., Aitia II. Avec ou sans Aristote. Le débat sur les causes a l'âge hellénistique et impérial, Editions Peeters SA, Leuven Belgium 2014.
} 
En los tratados de Aristóteles el término aitía aparece numerosísimas veces (casi 1000) y en varios pasajes Aristóteles distingue sus significados ${ }^{43}$. Pero en cualquier caso, Aristóteles parece dar una explicación general de la noción de causa, cuando introduce el concepto en el libro II de la Física en los siguientes términos: "Ahora que ha quedado esto delimitado, hay que investigar, sobre las causas, cuáles son y cuántas en número. Y puesto que el objeto de la investigación es conocer, y no creemos tener conocimiento sobre cada objeto hasta haber captado el «por

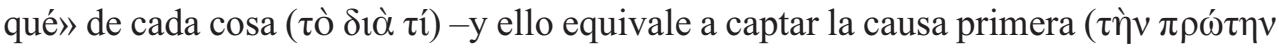
airíav)-, es evidente que también nosotros hemos de hacerlo sobre la generación y la destrucción y toda suerte de cambio natural, a fin de que, conociendo sus principios, intentemos conducir hacia ellas cada una de nuestras investigaciones" 44 .

Aristóteles pone de manifiesto que el porqué de cada cosa equivale a la causa primera. Por consiguiente, el conocimiento del "por qué" coincide con el conocimiento de la causa primera, como se confirma más adelante: "Es evidente, pues, que existen causas y que su número es, precisamente, el que hemos señalado, pues el

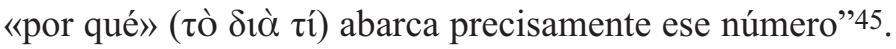

En los Analíticos segundos, Aristóteles identifica la causa con el término medio:

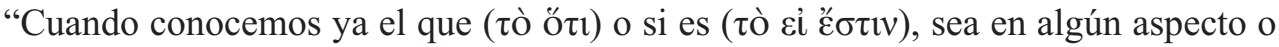

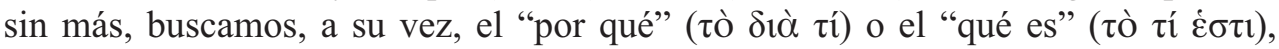

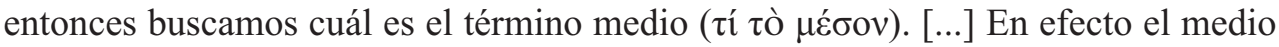

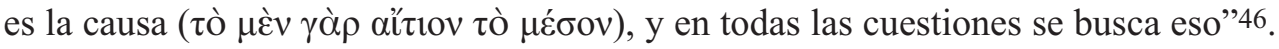

Este pasaje ha inducido a especialistas a identificar la causa en términos de "explicación". En este sentido, parece claro que las causas son cualquier respuesta que se dé a la pregunta ¿por qué?, es decir, cualquier forma de explicación de un fenómeno. Sin embargo, como se ha señalado, la identificación de la causa con el término medio no implica que todo término medio de un silogismo sea una causa (aition). Pues, como se sabe, para Aristóteles hay términos medios que no tienen función causal, mientras que otros sí47. En el pasaje previamente citado, Aristóteles está indicando que cuando tenemos ciencia de algo y buscamos el "por qué", la causa funciona como término medio en su silogismo correspondiente. Por consiguiente, su ser causa no depende de ser término medio, sino al contrario: ser término medio en un silogismo es lo que depende de su ser causa"48.

\footnotetext{
44 Aristóteles, Física, II, 3, 194b 16-23. Esta misma idea se repite en los Analíticos segundos, aunque en este lugar se hace referencia a la idea de causa necesaria: "Creemos que sabemos cada cosa sin más, pero no del modo sofístico, accidental, cuando creemos conocer la causa por la que es la cosa, que es la causa de aquella cosa y que no cabe que sea de otra manera". Aristóteles, Analíticos segundos, I, 2, $71 \mathrm{~b} 8-12$

45 Aristóteles, Física, II, 7, 198a 14-17.

46 Aristóteles, Analíticos segundos, II, 89b 38-90a 7. Cito por la traducción de Miguel Candel Sanmartín, en Aristóteles, Tratados de lógica (Órganon), vol II, Gredos, Madrid 1988.

47 Cfr. Aristóteles, Analíticos segundos, I, 78a 30-b 4.

48 Natali, Carlo, "Aitia in Plato and Aristotle. From everyday language to technical vocabulary", en C. Natali, C. Viano, M. Zingano (a cura di), AITIA I. Les quatre causes d'Aristote, pp. 57-58.
} 
En este sentido, y como se verá más adelante con mayor claridad, la causa en Aristóteles no es en sí misma una explicación, pues a través de estos discursos o de dichos conceptos, Aristóteles está aludiendo a "cosas", o sea, a realidades efectivamente existentes precedentemente, simultáneamente, o sucesivamente, externas o internas a la cosa. Además, Aristóteles habla de causas (en plural) y su significado no se reduce al concepto moderno de causa teorizado y criticado por ejemplo por David Hume 49 .

\section{Teoría de las cuatro causas}

Como se ha visto con anterioridad, en una primera fase, Aristóteles ha mostrado en el libro Alpha de la Metafisica que la sabiduría es una ciencia, es decir, conocimiento de ciertas causas y principios (cap. 1); en una segunda, ha dicho que tales causas y principios son primeros (cap. 2). Ahora, en esta tercera fase, Aristóteles pretende mostrar que las causas y los principios primeros son ni más ni menos que de cuatro tipos (cap. 3).

El capítulo tercero inicia así: "Es obvio, pues, que necesitamos conseguir la

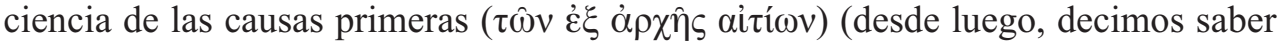

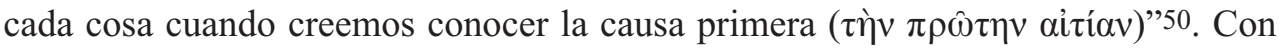
estas palabras, Aristóteles comienza el discurso sobre las causas primeras, pues, como ha dicho, conocer algo es conocer su causa primera. Aristóteles reconoce que todas las causas son principios, por lo que la expresión "primeros principios y causas" está referido a las causas primeras. En este sentido, la sabiduría es conocimiento de las causas primeras o de los principios, pues son términos equivalentes ${ }^{51}$.

E inmediatamente después, Aristóteles presenta la tabla completa de los diversos tipos de causa en los siguientes términos:

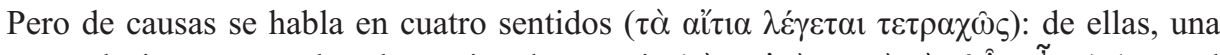
causa decimos que es la substancia o la esencia ( porqué se reduce, en último término, a la definición, y el porqué primero es causa y

49 Cfr. Hume, D., Tratado sobre la naturaleza humana, libro I, parte 3. La diferencia entre el concepto aristotélico y moderno de causa ha sido explicada por J. Follon en su artículo "Réflexions sur la théorie aristotélicienne des quatre causes", Revue philosophique de Louvain, 86 (1988), pp. 317-353 y por J. Lear en Aristotle: the desire to understand, Cambridge University Press, Cambridge 1988, pp. 30-31. En la reciente literatura de lengua española, Juan Arana en su reciente libro Los sótanos del universo: la determinación natural y sus mecanismos ocultos, Biblioteca Nueva, Madrid 2012, pp. 61101.

50 Aristóteles, Metafisica, I, 3, 983a 24-26.

51 Cfr. Aristóteles, Metafísica, V, 1, 1013a 17. La distinción entre causa y principio en el uso aristotélico es mínima y en la mayoría de los casos son términos sinónimos. Cfr. Ross, W.D., Metaphysics, vol. I, p. 291; Zanatta, Metafisica, p. 828. 


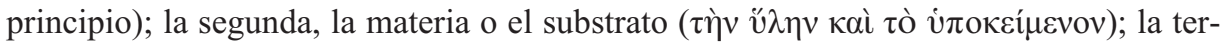

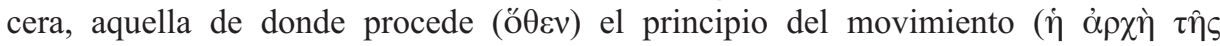

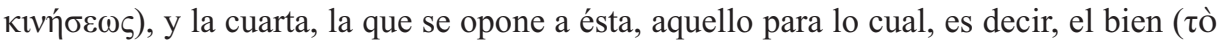

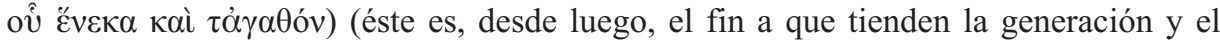
movimiento). 52

Con esta tabla, Aristóteles enumera sintéticamente los cuatro significados del término causa, recuperando su clasificación de las cuatro causas ya presente en el libro II de la Física, cuyo propósito consistía en ofrecer una explicación completa y exhaustiva del cambio53. En este lugar, Aristóteles escribió:

Pues bien, en un sentido se llama causa a aquello a partir de lo cual ( $\tau$ ò $\dot{\varepsilon} \xi$ oû) se origi-

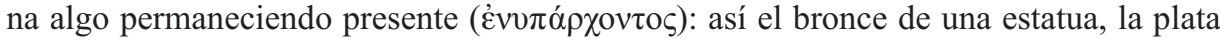
de una copa y sus géneros respectivos; en otro sentido se llama causa a la forma y al

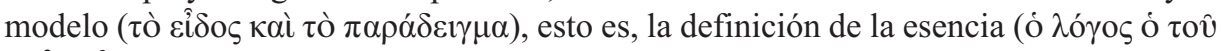

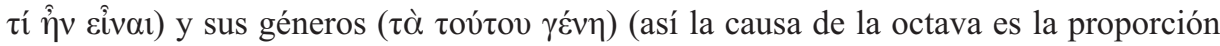
de dos a uno, y en general el número) así como las partes que entran en la definición. Aún más, causa es aquello de donde surge (ő $\theta \varepsilon v$ ) el inicio primero del cambio o del reposo: así, el que da un consejo es causa, el padre es causa del hijo y, en general, lo que hace es causa de lo que es hecho y lo que cambia es causa de lo que es cambiado. Más todavía, se habla de causa como el fin ( $\tau$ ò $\tau \varepsilon ́ \lambda o \varsigma)$, esto es, el aquello para lo cual ( $\tau$ ò oû

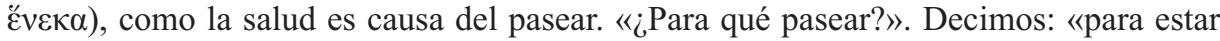
saludable» y en diciendo así creemos haber aducido la causa. ${ }^{54}$

En la tipología de las causas del texto de la Metafísica observamos que un primer tipo de causa es la substancia o el "qué era ser" (i் oủ el hecho de que el "por qué" se puede reconducir a su definición, a su lógos. Esta expresión forma una endíadis, es decir, un recurso retórico que presenta dos términos coordinados por una conjunción para referirse a un único concepto. El primer término es un sustantivo que proviene del participio presente del verbo ser, y que puede ser traducido como substancia, aún cuando este término no indica en todos los casos el "qué era ser"; el segundo término es una expresión acuñada por Aristóteles, que literalmente se podría traducir como el "qué era ser" (lat.: quod quid erat esse). Con esta expresión, Aristóteles se está refiriendo a la así denominada "causa formal", es decir, aquella que hace que algo sea lo que es, su forma (cî̉os), su esencia.

\footnotetext{
52 Aristóteles, Metafísica, I, 3, 983a 26-32.

53 Nadie anteriormente a Aristóteles había expuesto sistemáticamente todos los tipos de causas para ofrecer una explicación completa y exhaustiva del mundo de la naturaleza. Cfr. Falcon, Andrea, "Aristotle on Causality", The Stanford Encyclopedia of Philosophy (Fall 2011 Edition), Edward N. Zalta (ed.), URL $=<$ http://plato.stanford.edu/archives/fall2011/entries/aristotle-causality/>.

54 Aristóteles, Física, II, 3, 194b 24-36. Este elenco constituye también el contenido del capítulo 2 del libro $\Delta \mathrm{y}$ aparece también esta numeración en Analíticos segundos, II, 11.
} 
La esencia viene expresada mediante una definición, porque, indicando el género y la diferencia específica, permite establecer los confines (lat.: fines) en los que se encuentra lo que se define; por ejemplo: "animal" y "racional", en la definición de hombre, cuya causa formal está constituida por el alma racional55.

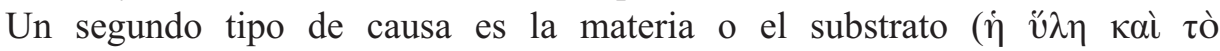

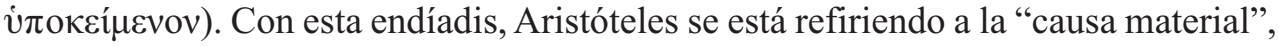
es decir, la materia de que algo está hecho y, en cuanto tal, puede ser identificada como el substrato, porque es lo que recibe la forma (lat.: substratum: aquello que está extendido por debajo); por ejemplo, el bronce es la causa material de una estatua o la plata de una copa ${ }^{56}$.

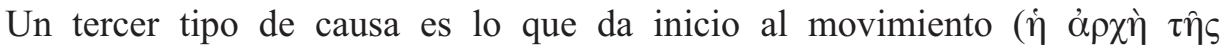

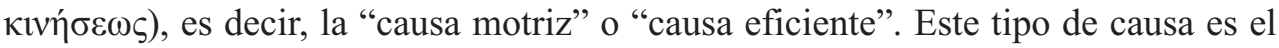
principio del movimiento, lo que hace que algo pase de un estado a otro diferente. Por ejemplo, el padre es la causa motriz del hijo ${ }^{57}$.

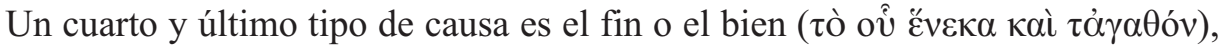
la así llamada "causa final". Este tipo de causa puede ser considerada como opuesta a la precedente, en el sentido en que, así como la causa motriz es "aquello de donde procede" el movimiento, la causa final es "aquello con vistas a lo que" se da el movimiento, esto es, el fin o la realización de eso. Aristóteles la identifica con el bien. Por ejemplo, la causa final de la salud es el pasear, pues se pasea con el fin de (para) tener salud 58 .

\section{La filosofía primera como ciencia de las causas primeras}

La exposición aristotélica de los cuatro géneros o tipos de causas apenas mencionados en la Metafisica no tiene un objetivo exclusivamente semántico, que establece en cuántos modos se dice el término "causa". Su objetivo es sobre todo gnoseológico y ontológico. Con los ejemplos que Aristóteles aduce para cada uno de los tipos de causas se pone de manifiesto que Aristóteles está aludiendo a "cosas", o sea, a realidades efectivamente existentes: el bronce respecto a la copa y la plata respecto a la copa es la causa material; la relación de dos a uno respecto a la octava y el número respecto a los acordes musicales es la causa formal; el que delibera

\footnotetext{
55 Cfr. Berti, E., Rossitto, C., Aristotele. Il libro primo della «Metafisica», pp. 71-72. Para explicar la traducción de estos términos como substancia y el "qué era ser" remito al texto inédito del prof. García Marqués, "Sentido y traducción de términos básicos de la Metafísica de Aristóteles", de inminente publicación.

56 Cfr. Berti, E., Rossitto, C., Aristotele. Il libro primo della «Metafisica», p. 72.

57 Cfr. Berti, E., Rossitto, C., Aristotele. Il libro primo della «Metafisica», p. 72.

58 Cfr. Berti, E., Rossitto, C., Aristotele. Il libro primo della «Metafisica», p. 72.
} 
respecto a una acción y el padre respecto al hijo es la causa motriz; la salud respecto del pasear es la causa final. Si la causa material o formal o motriz o final es un concepto funcional universal y, por tanto, vacío de por sí, éste se llena de una realidad particular que hace la función indicada. Por ello, Aristóteles ha indicado que las causas son universales, en cuanto al concepto, es decir, las mismas para todas las cosas, pero en relación a las realidades individuales son diferentes por especie y por número 59 .

Generalmente, eso es un aspecto muy importante que la mayoría de los intérpretes dejan de lado o no lo ponen de manifiesto suficientemente. Enrico Berti y Cristina Rossitto han indicado que para tener ciencia de un determinado objeto es necesario conocer las causas, y como las causas son de cuatro tipos, es preciso conocerlas todas; sólo así se alcanzará la explicación que justifica lo que sucede. En relación con un objeto, por tanto, será preciso preguntarse qué es, de qué está hecho, de dónde proviene, a qué tiende, tratando de responder a estas cuestiones diciendo cuál es su causa formal, su causa material, su causa eficiente y su causa final. Por ejemplo, habrá que explicar la especie "hombre" diciendo que el hombre es un animal racional, hecho de carne, huesos y músculos, generado por sus padres y con la tendencia a realizar su esencia propia, o sea, hacer uso de la razón. En cambio, una casa se explica diciendo que es un edificio correspondiente a un determinado proyecto, hecho de ladrillos, construido por los albañiles y con el objetivo de proteger de la intemperie60.

Por esta razón, inmediatamente después de enumerar los cuatro tipos o géneros diferentes de causas, el texto aristotélico prosigue del siguiente modo:

Aunque hemos tratado suficientemente de las causas en la Física, recordemos, sin embargo, a los que se dedicaron antes que nosotros al estudio de los entes y filosofaron sobre la verdad. Pues es evidente que también ellos hablan de ciertos principios y causas. Esta revisión será útil para nuestra actual indagación; pues, o bien descubriremos algún otro género de causa, o tendremos más fe en las que acabamos de enunciar. ${ }^{61}$

Aristóteles declara que ya se ha ocupado de los cuatro tipos de causa en general, en la Física. El objetivo siguiente consiste, por tanto, en averiguar cuáles son

59 Cfr. Berti, E., Aristotele. Dalla Dialettica alla filosofia prima, pp. 388-389. 391-393; Wieland, W., Die aristotelische Physik, pp. 202-230; Julia Annas, "Aristotle on Inefficient Causes", in The Philosophical Quarterly, vol. 32, no 129 (1982), pp. 311-326 (específicamente, pp. 319-323); Julius M. Moravcsik, "What Makes Reality Intelligible? Reflections on Aristotle's Theory of Aitia", in Lindsay Judson (ed.), Aristotle's Physics: A Collection of Essays, Clarendon Press, Oxford 1991, pp. 31-47. De la misma opinión es el citado libro de Juan Arana, Los sótanos del universo, pp. 71-73 y el reciente texto de Natali "Aitia in Plato and Aristotle. From everyday language to technical vocabulary", pp. 58-59.

60 Berti, E., y Rossitto, C., Aristotele. Il libro primo della «Metafisica», p. 72.

61 Aristóteles, Metafísica, I, 3, 983a 33 - 983b 6. 
las causas primeras conocidas por la sabiduría y examinar si éstas son exactamente, en número y por su naturaleza, del tipo que ya se han citado o si existen otras diferentes. Como han indicado una vez más, Berti y Rossitto, cada tipo o género de causa comprende muchas causas, concatenadas según un orden de prioridad, donde cada causa depende de la precedente y es causa de la sucesiva. De este modo, en la serie de las causas, la más cercana al objeto es aquella "próxima" y es "menos" causa, porque depende de otra causa superior a ella en la serie de las causas; la más lejana es aquella "remota", que es "más" causa que las demás, porque todas dependen de ésta, en cuanto que es primera de la serie: esta es, precisamente, la causa primera. Hay que investigar, por consiguiente, en cada uno de los cuatro diversos tipos o géneros de causas mencionados más arriba, cuál es la causa primera, o sea, la primera causa material, la primera causa formal, la primera causa eficiente y la primera causa final ${ }^{62}$.

La confirmación de que se deben buscar la causas primeras dentro de cada tipo o género de causa se encuentra en el libro alfa minúscula de la Metafisica, que concierne también este aspecto de la sabiduría como búsqueda de las causas primeras. Al final del capítulo primero, Aristóteles reconoce que los principios de los entes eternos no tienen una causa de su ser, sino que ellos son la causa del ser para las demás cosas ${ }^{63}$. Pero la exposición más clara de las causas primeras se encuentra en el capítulo segundo del mismo libro. En efecto, Aristóteles demuestra que, en el ámbito de cada uno de los géneros de causas descritos más arriba, no puede haber una serie infinita de causas y, por consiguiente, deben existir causas primeras: "Es evidente que hay algún principio, y que las causas de los entes no pueden ser infinitas ni en serie ni en cuanto a sus especies" 64.

Así pues, respecto a la causa material, se puede llegar hasta los elementos, es decir, agua, aire, tierra y fuego, que se transforman continuamente uno del otro. Cuando se conoce esta última causa material es preciso detenerse y afirmar que, en el ámbito de las causas materiales, las causas primeras son los elementos 65 . Respecto a la causa motriz o eficiente se puede llegar de las causas terrestres (por ejemplo el viento) a las causas celestes (por ejemplo el sol), y después a las causas de éstas últimas (por ejemplo, Empédocles creía que era el amor y el odio). Pero, en este momento hay que detenerse 66 . También, respecto a la causa final, se puede llegar, por ejemplo, en el caso del hombre, a la salud y de ésta a la felicidad. Pero es preciso detenerse aquí, porque si no se detiene en algún momento se suprime la misma existencia del fin y si ya no existe un fin nadie intentaría hacer nada ${ }^{67}$.

\footnotetext{
62 Berti, E., y Rossitto, C., Aristotele. Il libro primo della «Metafisica», p. 73.

63 Aristóteles, Metafísica, II, 1, 993b 29-30.

64 Aristóteles, Metafísica, II, 2, 994a 1-2.

65 Aristóteles, Metafísica, II, 2, 994a 3-5, a 19 - b 9.

66 Aristóteles, Metafísica, II, 2, 994a 5-8.

67 Aristóteles, Metafísica, II, 2, 994a 8-10, b 9-16.
} 
Finalmente, respecto a la causa formal, es decir, la que concierne a la definición, la primera es la más próxima, la más específica, y ésta es irreducible a otras definiciones más generales 68 .

En todos los casos, cuando se da una serie de causas, la primera es causa de todas las otras. Y si no hubiese una primera no habría ninguna causa. De este modo se demuestra que el proceso al infinito, en la serie de las causas, es imposible, ya que acabaría con la eliminación de la misma serie: "Si no hay ningún término primero, -declara Aristóteles- no hay en absoluto ninguna causa" 69 . Análogamente, como la ciencia es conocimiento de las causas, el proceso al infinito en la serie de las causas suprimiría cualquier posibilidad de hacer ciencia ${ }^{70}$. Demostrada la necesidad de que en cada género de causa deben existir causas primeras, a continuación quedará por investigar cuáles son esas causas primeras para que, de este modo, se pueda alcanzar la sabiduría.

\section{Una consideración final a modo de conclusión}

El libro I de la Metafisica de Aristóteles muestra el estatuto de una ciencia que investiga los principios y causas primeras. Como hemos visto, las causas pueden ser de cuatro tipos y, por consiguiente, esta ciencia debe investigar, en cada uno de los géneros, la causa primera71. Esta ciencia, que Aristóteles ha reconocido como "sabiduría", va a ser desarrollada en los libros sucesivos que forman parte de la Metafísica, caracterizándola también como ciencia del ente en cuanto ente (Libro Gamma), y/o ciencia teológica (Libro Épsilon).

En la actualidad tiene más éxito la concepción de la Metafisica como ontología, es decir, como un discurso que indaga la naturaleza última de todo lo que existe ${ }^{72}$, y en algunos ámbitos también se sigue entendiendo como teología racional ${ }^{73}$. Sin

\footnotetext{
68 Aristóteles, Metafísica, II, 2, 994a 10-11, b 16-20.

69 Aristóteles, Metafísica, II, 2, 994a 19.

70 Aristóteles, Metafísica, II, 2, 994b 20-23. Cfr. Berti, E., Aristotele. Dalla Dialettica alla filosofia prima, pp. 411-413.

${ }^{71}$ La definición y caracterización de la sabiduría como conocimiento de los primeros principios y causas no se encuentra de modo exclusivo en el libro primero de la Metafisica. Giovanni Reale en su monumental obra, Il concetto di filosofia prima e l'unità della Metafisica di Aristotele, ha presentado todos aquellos pasajes de la Metafísica de Aristóteles, haciendo notar que esta concepción de la sabiduría (dimensión etiológica, de aitia) se afirma de modo explícito en cada uno de los libros que componen esta obra. Reale, G., Il concetto di filosofia prima e l'unità della Metafisica di Aristotele, Vita e pensiero, Milán 1961 ( $6^{\mathrm{a}}$ ed. 1994).

72 Cfr. Tahko, Tuomas E., Contemporary Aristotelian Metaphysics, Cambridge University Press, Cambrige 2012.

73 Me refiero a autores como A. Plantinga, R. Swinburne, W. L. Craig o J. Polkinghorne. Sobre esta línea de investigación se puede consultar M. Micheletti, M., La teologia razionale nella filosofia analitica, Roma, Carocci, 2010.
} 
embargo, a mi modo de ver, y como se pone de manifiesto en el libro Alpha de la Metafísica, la filosofía primera en Aristóteles es ciencia de las causas primeras y éstas se constituyen como el verdadero objetivo de búsqueda, aquello que se debe encontrar al final de la investigación. Ciertamente, el objeto sobre el que versa esta

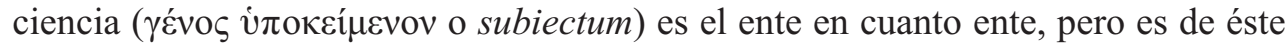
del que se deben investigar las cusas primeras y los principios. Por consiguiente, es el ente el que debe ser explicado, conociendo cuáles son sus principios y sus causas. De este modo, se pone de manifiesto el estatuto propio de la ciencia expuesto en los Analíticos segundos y otorga efectivamente a la filosofía primera un auténtico y genuino carácter científico, es decir, la constituye en ciencia. Pues, como declara Aristóteles, "toda ciencia discursiva, o que participe en alguna medida del pensar discursivo, se ocupa de causas y principios más exactos o más sencillos"74.

\section{Referencias bibliográficas}

Obras y traducciones de Aristóteles:

ARISTÓTeles, Analíticos segundos. Introducción, traducción y notas de Candel Sanmartín, M., en Aristóteles, Tratados de lógica (Órganon), vol II, Gredos, Madrid 1988.

ARIstóteles, Física. Introducción, traducción y notas de Calvo Martínez, J. L., CSIC, Madrid 1996.

ARISTóTEles, Metafísica. Introducción, traducción y notas de Calvo Martínez, T., Gredos, Madrid 1994.

REALE, G. Introduzione, traduzione e commentario della Metafisica di Aristotele, Bompiani, Milano 2004.

Ross, W. D. Aristotle's Metaphysics. A revised text with Introduction and Commentary, 2 vols., Oxford Clarendon Press, 1924. Reprinted lithographically, Oxford University Press, 1948, 1953 (with corrections) 1958.

Steel, C. Primavesi, O. (editors). Aristotle's Metaphysics Alpha, Oxford University Press, Oxford 2012.

ZanatTA, M. Aristotele, Metafisica, Introduzione, traduzione, BUR, Milano 2009.

Estudios:

AnNAS, J. "Aristotle on Inefficient Causes", in The Philosophical Quarterly, vol. $32, \mathrm{n}^{\circ} 129,1982$, pp. 311-326.

\footnotetext{
74 Aristóteles, Metafísica, VI, 1, 1025b 6-7. Cfr. Física, I, 1 184a 10-15.
} 
ARANA, J. Los sótanos del universo: la determinación natural y sus mecanismos ocultos, Biblioteca Nueva, Madrid 2012.

Aubenque, P. Le problème de l'être chez Aristote, Paris, 1962.

Bernardinello, S. Gli scoli alla "Metafisica» di Aristotele nel f. $234 \mathrm{r}$ del "Parisinus Graecus 1853» (E), in Moraux-Wiesner (herausgegeben), Zweifelhaftes im Corpus Aristotelicum, Studien zu einigen Dubia. Akten des 9. Symposium Aristotelicum, Walter de Gruyter, Berlin, 1983, pp. 39-54.

Berti, E., Rossitto, C. Aristotele. Il libro primo della «Metafisica», Laterza, Roma-Bari 1993.

Berti, E. Aristotele. Dalla Dialettica alla filosofia prima, Bompiani, Milano 2004. BERTI, E. "Note sulla tradizione dei primi due libri della «Metafisica» di Aristotele", en Elenchos, 3, 1982, pp. 5-38 (publicado también en: Nuovi studi aristotelici. II. Fisica, antropologia e metafisica, Morcelliana, Brescia, 2005, pp. 241-265.

Berti, E., Struttura e significato della Metafisica di Aristotele, Edusc, Roma, 2006. Bouygues, M. Averroès, Grand Commentaire de la Métaphysique, Beyrouth, 1952. Broadie, S. "A Science of First Principles", en Steel, C. Primavesi, O. (editors). Aristotle's Metaphysics Alpha, Oxford University Press, Oxford, 2012, pp. 4367.

Calvo Martínez, J. L. "Introducción” a Aristóteles, Física, CSIC, Madrid, 1996, pp. XI-CV.

Cambiano, G. "The desire to know" en Aristotle's Metaphysics Alpha, Oxford University Press, Oxford, 2012, pp. 39-42.

Darms, G. Averroes, In Aristotelis librum II Metaphysicorum Commentarius, Freiburg-Schweitz, 1966.

Drossaart Lulofs, H. J. Nicolaus Damascenus on the Philosophy of Aristotle, E. J. Brill, Leiden, 1969.

FAlcon, A. "Aristotle on Causality", en The Stanford Encyclopedia of Philosophy, Edward N. Zalta (ed.), [soporte electrónico], 11 enero 2006 (revisión 15 octubre 2012) < http://plato.stanford.edu/archives/fall2011/entries/aristotle-causality/>. [20 noviembre 2014].

Follon, J. "Réflexions sur la théorie aristotélicienne des quatre causes", Revue philosophique de Louvain, 86, 1988, pp. 317-353.

JAEGER, W. Aristoteles, de la versión española de José Gaos Aristóteles: Bases para la historia de su desarrollo intelectual, FCE, México, 1946.

LEAR, J. Aristotle: the desire to understand, Cambridge University Press, Cambridge, 1988.

LÓPEZ MolinA-NiÑIROLA, J.M. "Recepción y significado del uso científico de la dialéctica aristotélica”, Pensamiento. Revista de Investigación e Información, vol. 71, n. 269 (2015), pp. 1261-1276. 
MANSION, A. "Introduction" à la physique aristotélicienne, Publications Universitaires de Louven, Lovain-Paris, 1945.

Micheletti, M., La teologia razionale nella filosofia analitica, Roma, Carocci, 2010.

MunK, S. Mélanges de philosophie juive et arabe, Vrin, Paris, 1955.

Moraux, P., Wiesner. J. (ED.). Zweifelhaftes im Corpus Aristotelicum. Studien zu einigen Dubia. Akten des 9. Symposium Aristotelicum, Walter de Gruyter, Berlin, 1983.

MoraVCSIK, J. M. "What Makes Reality Intelligible? Reflections on Aristotle's Theory of Aitia", in Lindsay Judson (ed.). Aristotle's Physics: A Collection of Essays, Clarendon Press, Oxford, 1991, pp. 31-47.

NAtALI, C. "Aitia in Plato and Aristotle. From everyday language to technical vocabulary", en Natali, C. Viano, C. Zingano, M. (a cura di), AITIA I. Les quatre causes d'Aristote. Origines et interprétation. Leuven, Editions Peeters SA, Leuven Belgium, 2013, pp. 57-58.

Natali, C. Viano, C. Zingano, M. (a cura di). Aitia I. Les quatre causes d'Aristote. Origines et interprétation. Leuven, Editions Peeters SA, Leuven Belgium, 2013.

Natali, C. Viano. Aitia II. Avec ou sans Aristote. Le débat sur les causes a l'âge hellénistique et impérial, Editions Peeters SA, Leuven Belgium, 2014.

Primavesi, O. "Aristotle, Metaphysics A. A New Critical Edition with Introduction”, en Aristotle's Metaphysics Alpha, Oxford University Press, Oxford, 2012, pp. 385-516.

ReAle, G. Il concetto di filosofia prima e l'unità della Metafisica di Aristotele, Vita e pensiero, Milán, 1961 ( $6^{a}$ ed. 1994).

Tahko, T. E., Contemporary Aristotelian Metaphysics, Cambridge University Press, Cambrige, 2012.

Vuillemin-Diem, G. Anmerkungen zum Pasikles-Bericht und zu Echtheitszweifeln am grösseren und kleineren Alpha in Handschriften und Kommentaren, in Moraux-Wiesner (herausgegeben), Zweifelhaftes im Corpus Aristotelicum. Studien zu einigen Dubia. Akten des 9. Symposium Aristotelicum, Walter de Gruyter, Berlin, 1983, pp. 157-192.

Wieland, W. Die aristotelische Physik, Vandenhoeck \& Ruprecht, Göttingen, 1970.

José Antonio García-Lorente

Universidad de Murcia

garcia-lorente@um.es 